\title{
OIL EXTRACTS OF WILD APPLE FRUIT AS ACTIVE SUBSTANCES IN UV PROTECTION PREPARATIONS
}

\author{
Dragana Stojiljkovićc ${ }^{* *}$, Ivana Arsićc ${ }^{2}$, Vanja Tadić3 \\ ${ }^{1}$ Health Care Institution Pharmacy "Farmakop", Niš, Serbia \\ 2 Department of Pharmacy, Faculty of Medicine, University of Niš, Niš, Serbia \\ 3 Department for Pharmaceutical Research and Development, Institute for Medicinal Plant Research \\ "Dr Josif Pančić", Belgrade, Serbia
}

\begin{abstract}
Permanent skin exposure to the sun may cause a lot of side effects due to the action of UVA and UVB rays. Several studies have shown that polyphenols inhibit the reactive oxygen species' formation as their "scavengers", reduce the penetration of $U V B$ rays to sensitive tissues, neutralize free radicals, and because of their antioxidant and anti-inflammatory properties, participate in the prevention and treatment of many diseases caused by UVB rays. The use of cosmeceuticals rich in polyphenolic compounds represents a good basis for health improvement and prevention of UV-related chronic diseases. Apple fruit contains a large number of biologically active antioxidant substances, and their extracts can be potentially used for the prevention and/or treatment of many skin diseases caused by oxidative stress. The aim of our study was to estimate the antioxidant activity of wild apple fruit oil extracts obtained by different extraction methods with virgine olive and sunflower oil as solvents in order to investigate the possibility of their use as active substances with antioxidant effects in dermocosmetic/cosmetic UV skin protection preparations. Two extraction methods were applied. Method 1 comprised the maceration of the wild apple fruit in olive or sunflower oil on a water bath for 4 hours (Samples S1 and S2). Method 2 comprised the maceration of dry wild apple fruit in 96\% ethanol followed by maceration with the olive or sunflower oil extraction, heating up on the water bath and removing the ethanol throughly at same time ( $S_{3}$ and $\left.S_{4}\right)$. All extracts were prepared in the drug:extract ratio of 1:5. Total phenolic content (TPC) was determined by Folin-Ciocalteu method and expressed as mgGAE/1oog d.w. Antioxidant activity was determined by two methods: DPPH test and test with linoleic acid and expressed as \%RSC (Radical Scavening Capacity) and \%AOA (AntiOxidant Activity), respectively. The type of used solvent did not have a significant influence on TPC and the antioxidant activity of oil extracts, but the used method of extraction was an important factor. TPC was the best for S4 (996.49 mgGAE/10og d.w.). S3 showed good TPC, too (729.9O mgGAE/1Oog d.w.). \%RSC of S1 and S2 were $14.94 \%$ and $18.11 \%$, and \%AOA were $62.20 \%$ and $61.21 \%$, respectively. The use of $96 \%$ ethanol additionally increased the antioxidant capacity ( $S_{3}$ and $S_{4}$ ), compared to the extraction with oil solvents only (more in DPPH test). \%RSC of $S_{3}$ and $S_{4}$ were $20.63 \%$ and $35.20 \%$, and \%AOA were $63.48 \%$ and $66.48 \%$, respectively. All extracts showed a higher ability to prevent lipid peroxidation, and a lower one to neutralize free radicals. Emulsions with oil extract showed satisfactory antioxidant activity. Oil extracts of wild apple fruit have good antioxidant possibilities and they can be used as active substances for UV protection.
\end{abstract}

Key words: Wild apple fruit, oil extracts, antioxidant activity

\section{INTRODUCTION}

Oxidative stress (OS) is an imbalance between the cellular production of reactive oxygen species (ROS) and cells ability for their removal. One of the most important sources of OS, ultraviolet (UV) radiation, can induce the production of ROS, which can react with a large number of potential target molecules in the skin [1], [2]. Permanent skin exposure to sunlight may cause the damage of structure and function of skin by inducing a number of biological responses, including the development of wrinkling, scaling, dryness, mottled pigment abnormalities such as hypo- and hyperpigmentation, erythema and edema, sunburn cell formation, hyperplasia, photoaging and melanogenesis, and it is necessary to reduce or prevent these adverse effects on the skin with an additional defense [1]-[3].

UVA radiation penetrates through epidermis, dermis and reaches to subcutaneous tissues. At the cellular level, mainly UVA radiation causes OS, in which ROS play the main role. As a result of this stress, cells react to UVA damage with modified gene expression, which eventually results in abnormal cell morphology, cell apoptosis or necrosis, aging and skin damages. UVB leads to the high level of ROS formation in the skin, resulting in photooxidative damage of the cells and the extracellular matrix [1]-[4]. ROS generated by UV radiation may lead to lipid peroxidation, damage of membrane proteins and deoxyribonucleic acid mutations, alteration of the immune system, which can lead to various structural, functional and aesthetic changes on the skin, as well as to the development of many diseases. All biological systems possess an antioxidant defense system, which protects from OS, and if this defense system fails, damages are larger [2], [5].

Increased consumer demands for a safe and secure application of oral and topical products rich in polyhenols lead to a greater need for the search of new

"The paper was presented at the Fourth International Conference on Radiation and Applications in Various Fields of Research (RAD 2016), Niš, Serbia, 2016.

**s.dragana83@yahoo.com 
sources of natural antioxidants to replace synthetic ones. Topical application of sunscreen preparations with natural antioxidant compounds (primarily polyphenols) is very important for the strengthening of the protective endogenous system, as well as, in skin prevention and/or protection from UV radiation, ROS and OS influences [1].

Polyphenols are the most important active substances (AS) from plants, and they inhibit the ROS formation as their "scavengers", reduce the penetration of UVB rays to sensitive tissues and neutralize free radicals. Hence, possessing the antioxidant and antiinflammatory properties, they might participate in the prevention and/or treatment of many diseases, including disorders caused by OS and cell aging [5]. Polyphenols can act as physical barrier preventing the penetration of UV light into the skin and absorbing the entire UVB spectrum and part of the UVA and UVC range. Polyphenols have the potential to protect the skin against the adverse effects of UV light. Chemoprevention with natural antioxidants is an option for the protection against sunlight-related skin disorders. All these effects of polyphenols are known as the sunscreen effects [6], [7].

The development of natural products is especially pronounced in the area of dermocosmetic/cosmetic preparations, which should, in addition to stability, efficiency and safety, contain the raw materials of "natural" origin (plant extracts, emulsifiers...) and without parabens, paraffin, silicone, to meet the sophisticated requirements of modern consumers [8]. When polyphenols are applied topically on the skin, the sunscreen effect contributes to the photoprotective effects by reducing inflammation, OS and immunosuppression [7]. Some authors have shown that phenols from plants could be acceptable in topical preparations, particularly in facial sunscreen creams [3]. Also, formulations containing extracts of different plants with polyphenols may provide substantial photoprotective effects because different aspects of skin damage are affected by each extract [3]. Despite the increased public awareness of the dangers of solar UV exposure, the incidence of melanoma and nonmelanoma skin cancers continues to rise at an alarming rate. Natural antioxidant compounds with cell repair potential are promising agents for sun protection. Considering that UV induces OS-mediated adverse effects in the skin, the regular intake or topical application of polyhenols is suggested to be a useful way to reduce the harmful effects of UV radiation [2].

For this study, wild apple (Malus sylvestris (L.), Mill., Rosaceae) from the territory of Serbia was selected. The investigated wild apple represents a biomarker of the territory of Serbia, and a plant used in Serbian folk medicine for the treatment of various diseases caused by OS and ROS, such as hypertension, atherosclerosis, diabetes, rheumatism, urinary infections, for phototoxic skin changes, eczema, dermatitis, as well as raw material for potential use for the production of cosmetic/dermocosmetic sunscreen preparations [5], [9], [10]. This plant material was no further investigated as a source of active substances (AS) that can be used in the formulation of effective and safe sunscreen preparations. Wild apple fruit (WAF) contains variety of substances (polyphenolic compounds) with antioxidant activity (AA), and the content of these compounds in extracts depends on the 188 quality of the fruit as a drug, used solvents and extraction methods [5]. These investigations are necessary to define the optimal conditions of extraction and to obtain isolates with desired characteristics, for further utilization as AS and incorporation into preparations intended for topical use. Extraction by several solvents provides better separation and extraction of polyphenolic compounds [11]. The use of $96 \%$ ethanol in extraction with several solvents, one after another, can additionally increase the content of the extracted AS in the extract, and thus the AA of these extracts, wherein the ethanol is subsequently removed by evaporation.

A larger number of plant materials contain a certain amount of AS that show AA and moisturizing effect, so in plant raw material we can talk about the potential synergism of the AS [12], [13]. The use of various vegetable oils is more present in dermocosmetic industry, because they are good solvents for the extraction of AS from plant raw materials, and they are compatible with skin; additionally, they show positive effects on the skin (e.g. moisturizing effect), and have good pharmaceuticaltechnological and cosmetic characteristics [5], [14]. For this study, virgine olive and sunflower oil were selected as solvents. Oil extracts incorporated into the emulsion might additionally demonstrate the moisturizing effect on the skin, and can influence the emulsion structuring [13]-[15].

Substrates commonly used for cosmetic and dermocosmetic sunscreen preparations are usually stabilized by traditional ionic and non-ionic emulsifiers, which can cause the skin barrier function damage and/or skin irritation. Unlike them, non-ionic mixed emulsifiers of natural origin from the group of alkyl polyglucoside (so-called "green surfactants"), are biodegradable, made from natural raw materials, and have a dermatological favorable profile [16], [17].

The aim of our study was to estimate the content of polyphenols, as the most important compounds, and AA of oil extracts of wild apple fruit (OEWAF) obtained by maceration and digestion with virgine olive oil and sunflower oil as solvents in order to investigate the possibility of their use as AS with antioxidant effects in UV skin protection preparations. Extraction of bioactive compounds from WAF, determination of total phenolic content (TPC) and AA of made extracts, their incorporation into the emulsion stabilized by conventional and new, natural (biodegradable) emulsifiers and in vitro investigation of AA of these emulsions, is a way to scientifically verify the correctness of the traditional application of WAF as AS in certain skin conditions and to formulate safe and effective UV protection preparation.

\section{MATERIAL AND METHODS}

\subsection{Materials}

\subsubsection{Chemicals}

Virgine olive oil was from Medsol supplier (Molfetta (BA), Italy), sunflower oil from Sunce (Sombor, Serbia) and 96\% ethanol (v:vvolume:volume) were purchased from Hemos 
D. Stojiljković et al., Oil Extracts of Wild Apple Fruit..., Rad. Applic., 2016, 1, 3, 187-192

(Belgrade, Serbia). Folin-Ciocalteu reagent (FCR), DPPH (1,1-diphenyl-2-picryl-hydrazyl) radical, linoleic acid, BHT (butylated hydroxytoluene) and gallic acid were purchased from Sigma-Aldrich (USA). Sodium carbonate( $\left.\mathrm{Na}_{2} \mathrm{CO}_{3}\right)$, hydrochloric acid ( $\left.\mathrm{HCl}\right)$, iron(III) chloride $\left(\mathrm{FeCl}_{3}\right)$, phosphate buffer, ammonium thiocyanate, methanol and Polysorbates 20 were purchased from Centrohem doo (Stara Pazova, Serbia). Montanov ${ }^{\mathrm{TM}} 82$ and Montanov ${ }^{\mathrm{TM}} 14$ was from Seppic supplier, France and Emulgade SE from Cognis, Germany.

\subsubsection{Plant material}

Wild apple fruit (Malus sylvestris (L.) Mill., Rosaceae) was collected in September 2012 on the slopes of Kopaonik Mount (village Perunika) - southern Serbia. Seeds were removed from the fruits, fruits were cut into thin slices (degree of fragmentation was $5 \mathrm{~mm}$ ) and air-dried at $22 \pm 2^{\circ} \mathrm{C}$, for three weeks. Before extraction, drug was pulverized for 2 min using a mill (IKA - Werke, Staufen, Germany) and drug degree of fragmentation was reduced to $1 \mathrm{~mm}$.

\subsection{Extraction methods}

Oil liquid extracts of wild apple fruit were prepared with two solvents, virgine olive oil and sunflower oil by two extraction methods, maceration and digestion, according to the procedure of Pharmacopoeia of Jugoslavica, where extraction procedures are exactly described [18], versus the latest international pharmacopoeias. All extracts were made in drug:extract ratio 1:5 (w:v - weight:volume), stored and used for further investigation as liquid extracts.

\subsubsection{Maceration with oil}

The dried powdered WAF (20g) was macerated with 5 parts of oil on a water bath for $4 \mathrm{~h}$, in a tightly closed erlenmeyer flask, protected from direct sunlight, at $45 \pm 2{ }^{\circ} \mathrm{C}$, with occasional shaking. Oil extract was separated from the drug by straining and subsequent pressing and then it was filtered (Samples S1 and S2).

\subsubsection{Digestion with oil}

The dried powdered WAF (20g) was macerated with 3 parts of $96 \%$ ethanol for $24 \mathrm{~h}$, in a tightly closed erlenmeyer flask, protected from direct sunlight, at $22 \pm 2^{\circ} \mathrm{C}$, followed by maceration with 5 parts of oil extraction, in a water bath for $4 \mathrm{~h}$, at $45 \pm 2^{\circ} \mathrm{C}$, with occasional shaking, with removing ethanol thoroughly at the same time. Oil extract was separated from the drug by straining and subsequent pressing and then it was filtered (Samples $\mathrm{S}_{3}$ and $\mathrm{S} 4$ ).

\subsection{Formulation of emulsions with $O E W A F$}

Two emulsions of o/w type were made, with the same complex composition of the oil phase by varying the emulsifier (biodegradable - Montanov ${ }^{\mathrm{TM}}$ and conventional - Emulgade SE) and with liquid OEWAF, as the active substance ( $\mathrm{S} 4$ - extract made by digestion and sunflower oil as solvent), in a concentration of $6 \%$. Two placebo samples were made, too.

Emulsion EMDS and placebo PLM were made using biodegradable emulsifiers (Montanov ${ }^{\mathrm{TM}} 82$ and Montanov $\left.{ }^{\mathrm{TM}} 14\right)$. EEDS emulsion and placebo PLE were stabilized with a conventional emulsifier (Emulgade SE) (Table 1).

Table 1. Qualitative and quantitative composition of the examined emulsions and placebo

\begin{tabular}{|l|c|c|c|c|}
\hline \multirow{2}{*}{ Components } & PLE & EEDS & PLM & EMDS \\
\cline { 2 - 5 } & $\% \mathrm{~m} / \mathrm{m}$ & $\% \mathrm{~m} / \mathrm{m}$ & $\% \mathrm{~m} / \mathrm{m}$ & $\% \mathrm{~m} / \mathrm{m}$ \\
\hline Oil phase & 21.5 & 21.5 & 24.5 & 24.5 \\
\hline Water phase & 78.5 & 72.5 & 75.5 & 69.5 \\
\hline $\begin{array}{l}\text { Oil extract of } \\
\text { wild apple fruit }\end{array}$ & - & 6.0 & - & 6.0 \\
\hline
\end{tabular}

Samples of o/w type emulsions were prepared using standard procedures for making a hydrophilic emulsion with the same composition of water and oil phase, with OEWAF (S4), and with variation of emulsifier as a potential stabilizer. Investigated and placebo samples were prepared by heating and stirring in a closed glass vessel, wherein the oil phase, together with the emulsifier, was heated on thermostatic heating plate of a magnetic stirrer (magnetic stirrer IKAMAG (IKA, Germany)) at $70^{\circ} \mathrm{C}$ and then added to the water phase, already heated at $72^{\circ} \mathrm{C}$ with stirring (propeller rotary laboratory stirrer RW16 basic (IKA®Werke, Germany)) at a constant temperature for the following $5 \mathrm{~min}(800 \mathrm{rpm})$ and then for $3 \mathrm{~min}$ (500 $\mathrm{rpm})$. At $40^{\circ} \mathrm{C}$, the extract (S4) was added and cooling (with stirring at $300 \mathrm{rpm}$ ) was continued until reaching room temperature [19], [20].

\subsection{Method for the determination of TPC}

Total phenolic content (TPC) in oil extracts was determined by the colorimetric method using FolinCiocalteu reagent (FCR) [21], with minor modifications. Briefly, the reaction mixture was made by mixing $0.1 \mathrm{~mL}$ of investigated liquid extract, $7.9 \mathrm{~mL}$ of distilled water, $0.5 \mathrm{~mL}$ of FCR and $1.5 \mathrm{~mL}$ of $20 \%$ solution of $\mathrm{Na}_{2} \mathrm{CO}_{3}$. After $2 \mathrm{~h}$ of standing at $22 \pm 2^{\circ} \mathrm{C}$, the absorbance was measured at $765 \mathrm{~nm}$ using Evolution 60 UV/VIS spectrophotometer (Thermo Fisher scientific, USA). The results were expressed as milligrams of gallic acid equivalents per $100 \mathrm{~g}$ of dry plant material (mg GAE/10o g d.w. - dry weight).

\subsection{Methods for the determination of $A A$}

\subsubsection{Radical-scavenging activity}

To determine Radical Scavenging Capacity (RSC) of the investigated samples (OEWAF and emulsions with OEWAF), DPPH assay was used [22], with minor modifications. In the determination of $\mathrm{AA}$ of made emulsions with OEWAF, emulsions were first diluted with a mixture of Polysorbate 20 and water 1:5, w/w [20]. The ratio of emulsion mass and made mixture was $1: 3(\mathrm{w} / \mathrm{w})$. After dilution, the samples were mixed by rotary stirrer for $15 \mathrm{~min}$ at $400 \mathrm{rpm}$, at $50^{\circ} \mathrm{C}$. In this way "stock" solution was obtained, which was used in the further process of determination of the AA of emulsions. Briefly, $100 \mu \mathrm{L}$ of investigated samples (extracts and "stock" solutions of emulsions) was mixed with $900 \mu \mathrm{L}$ of fresh $0.04 \mathrm{mg} / \mathrm{mL}$ methanol solution of DPPH. Sample and blind solution (solution without sample) were incubated for 2omin in dark, at $22 \pm 2^{\circ} \mathrm{C}$, until stabilization. The absorbance was read at $517 \mathrm{~nm}$ using a spectrophotometer, with methanol as 
the reference solution. Results were expressed as percentage inhibition of DPPH reduction, i.e. as \%RSC. The synthetic antioxidant BHT was used as a positive control. The percentage of inhibition, i.e. \%RSC was calculated using the following equation:

$$
\% \mathrm{RSC}=[(\text { Asp - As }) / \text { Asp }] \times 100
$$

\%RSC - percentage of RSC, i.e. percentage of inhibition,

Asp - absorbance of the blind solution,

As - the absorbance of the sample solution.

\subsubsection{Inhibition of linoleic acid oxidation}

To determine the extract's ability to inhibit lipid peroxidation, linoleic acid and thiocyanate method was used, with a minor modification [23]. This assay was used to determine only the AA of oil extracts. Briefly, $0.5 \mathrm{~mL}$ of linoleic acid solution was mixed with $0.5 \mathrm{~mL}$ of $0.05 \mathrm{M}$ phosphate buffer $\left(\mathrm{pH}_{7}\right)$, then $100 \mu \mathrm{L}$ of the investigated oil liquid extract (D:E $=1: 5)$ and $150 \mu \mathrm{L}$ of distilled water were added to obtain a mixture which was incubated. After incubation $\left(24 \mathrm{~h}, 40 \pm 2^{\circ} \mathrm{C}\right), 100$ $\mu \mathrm{L}$ of incubated mixture was mixed with $9.7 \mathrm{~mL}$ of $75 \%$ methanol, $100 \mu \mathrm{L}$ of $30 \%$ ammonium thiocyanate, and $100 \mathrm{~mL}$ of $0.02 \mathrm{M}$ iron(III)chloride in $3.5 \mathrm{~mL}$ of $\mathrm{HCl}$. The same procedure was performed without incubation. Absorbance was read at $500 \mathrm{~nm}$. Results were expressed as \%AOA. AntiOxidant activity (AOA), i.e. the level of linoleic acid peroxidation was calculated using the following equation:

$$
\% \mathrm{AOA}=[(\Delta \mathrm{Ac}-\Delta \mathrm{As}) / \Delta \mathrm{Ac}] \times 100 .
$$

\%AOA - percentage of antioxidant activity, $\Delta \mathrm{Ac}$ - the difference in absorbance of control (mixture without sample) with/or without incubation,

$\Delta$ As - the difference in absorbance of sample with/or without incubation.

\subsection{Statistical analysis}

All results were expressed as the arithmetic mean of three independent measurements \pm standard deviation (SD). The TPC in OEWAF and AA of these oil extracts and emulsions with sunflower oil extract in correlation to the applied solvents and extraction methods, were analysed by analysis of variance, (ANOVA), followed by Tukey's t-test, with statistical significance of ${ }^{*} p<0.05$.

\section{Results and discussion}

\subsection{Analysis of TPC in OEWAF}

Polyphenolic compounds have a therapeutic effect on various diseases, and their topical application can have sunscreen effects on skin [2]. Some polyphenols are bound, so they cannot be identified and extracted using only one method or solvent, and it is necessary to apply different solvents and extraction methods [5].

TPC in OEWAF was the lowest for S2 (172.91 mgGAE/10og d.w.) and the best for $\mathrm{S}_{4}$ (996.49 mgGAE/10og d.w.). S3 showed good TPC and better than S1 (729.90 mgGAE/100g d.w. and 400.47 mgGAE/10og d.w., respectively) (Fig.1). The use of $96 \%$ ethanol additionally increased the content of phenols (almost twice), compared to the extraction with oil solvents only, and digestion showed the best content of phenolic compounds.

The polyphenols from WAP represent an extremely interesting and promising group of molecules. Their skin photoprotective elements have sun screening, antiinflammatory and AA that remove or reduce UV-induced damage. In our study oils were good solvents and gave good content of phenols, but oils can also bind polyphenols, and in that case, phenols sometimes cannot show their good AA [5].

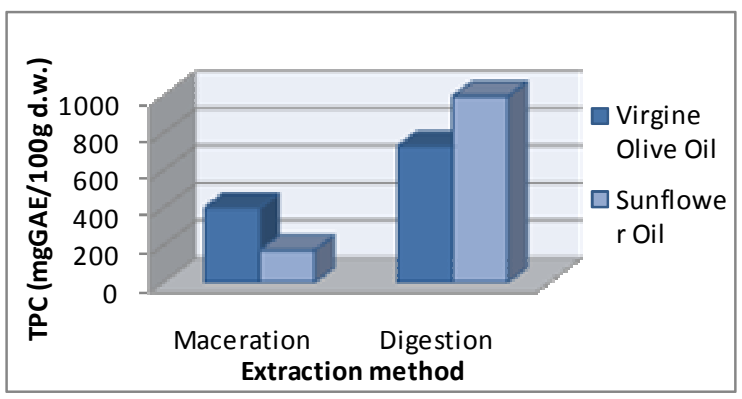

Figure 1. Total Phenol Content in OEWAF

\subsection{Analysis of $A A$ of $O E W A F$}

In complex systems, such as natural extracts, with different polyphenolic components it is useful to make a comparative examination of AA by different methods. Therefore, in our research we applied two spectrophotometric assays, different in their working principles. The AA of tested OEWAF was investigated by radical scavenging activity determination, using the DPPH method, and the ability to prevent lipid peroxidation was determined using linoleic acid. Antioxidants from the WAF react with a free DDPH radical and thus interrupt the oxidation chain reaction by donating hydrogen from the hydroxyl group of polyphenols, and provide a stable final product preventing oxidation [24]. The test with linoleic acid might be suitable for the assessment of prevention of lipid peroxidation and this method should be best suited for oil extracts. Results of DPPH assay were presented as \%RSC and of assay with linoleic acid as \%AOA. In both assays, there is a direct correlation, i.e. higher values indicate higher antioxidant activity.

\%RSC of S1 and S2 were $14.94 \%$ and $18.11 \%$, and \%AOA were $62.20 \%$ and $61.21 \%$, respectively. The use of $96 \%$ ethanol additionally increased the antioxidant capacity ( $\mathrm{S}_{3}$ and $\mathrm{S}_{4}$ ), compared to the extraction with oil solvents only (more in DPPH test). \%RSC of S3 and $\mathrm{S} 4$ were $20.63 \%$ and $35.20 \%$, and \%AOA were $63.48 \%$ and $66.48 \%$, respectively. All extracts showed better ability to prevent lipid peroxidation, and lower to neutralize free radicals. The differences in AA between extracts were higher in DPPH test, than in linoleic acid assay, where all extracts showed similar results. Because of that, only the DPPH test was used for the analysis of AA of emulsions with oil extract. The best result was shown by $\mathrm{S} 4$ (extract made by digestion using sunflower oil as solvent) in both tests (Fig. 2 and Fig. 3). Oil extracts showed better AA using the test with linoleic acid versus DPPH test, as we expected. 


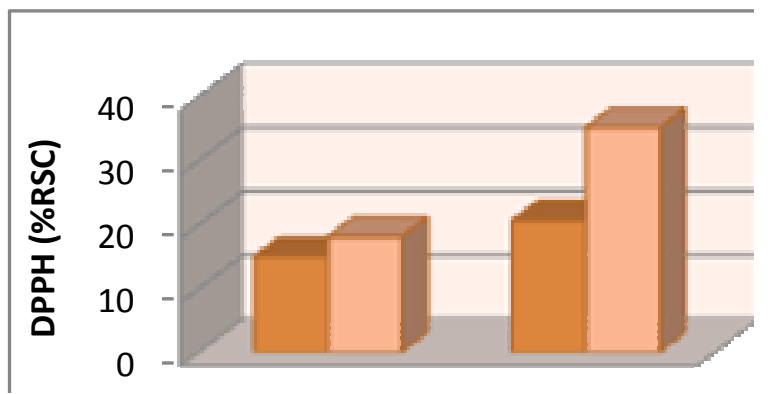

Figure 2. Radical Scavenging Activity of OEWAF

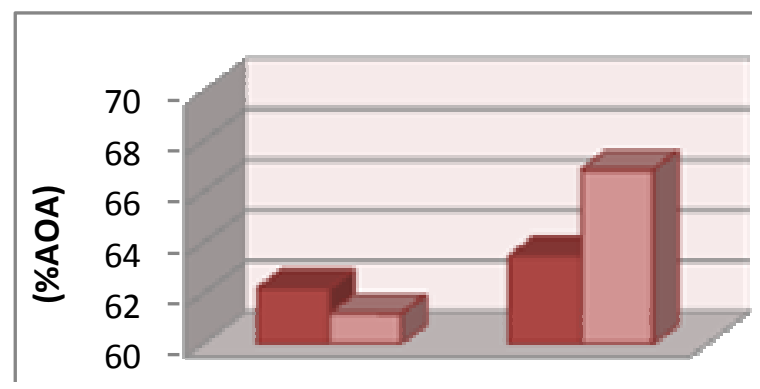

Figure 3. AntiOxidant Activity of OEWAF

Cekic and Ozgen [25] showed that wild cultures have better AA than the cultivated ones (using DPPH test). A good AA of wild apple fruit extracts using linoleic acid was shown too, where results were higher than our results [26]. Statistically significant difference was not found in AA of investigated extracts in relation to applied extraction methods, but in our study, digestion generally was the better method, because the extracts prepared by this method ( 33 and $\mathrm{S}_{4}$ ) had generally higher AA compared to the extracts made by maceration and using the same solvent ( $\mathrm{S} 1$ and S2). Digestion was a better method for AA, compared to maceration, probably because of ethanol, as co-solvent. Comparing the $\mathrm{AA}$ of investigated extracts in relation to using solvents, the analysis showed that the type of used solvent did not have a significant influence on AA of OEWAF, but the use of $96 \%$ ethanol in the digestion, as an extraction method, improved AA of oil extracts in both tests, twice more using DPPH test.

Oils as solvents are good for extraction of phenols from WAF, but at the same time, oils are good emollients for the skin [13]-[15]. Because of that, OEWAF might be, at the same time, active antioxidant substances and active moisturizing substances. Preparations with these extracts might have antioxidant and moisturizing properties, and might be used for the prevention and/or treatment of skin UVrelated-diseases.

\subsection{Analysis of $A A$ of emulsions with $O E W A F$}

OEWAF showed satisfactory AA. Because of that, in vitro investigation of efficiency, i.e. of AA of the emulsion was carried out in order to estimate the possible application of oil extracts of wild apple fruit from the territory of Serbia as AS in dermocosmetic/cosmetic UV protection preparations. Extract obtained by digestion with sunflower oil as solvent showed the best activity in both tests, and this extract ( $\mathrm{S} 4)$ was incorporated in the emulsion for the further estimation of AA of emulsions with OEWAF and was stabilized by conventional and natural, biodegradable emulsifiers. The AA was evaluated by investigation of the emulsion ability, i.e. of OEWAF as AS in emulsions, to act as "scavengers" of ROS and for this purpose only DPPH assay was used. Polyphenols from OEWAF react with DPPH radicals and interrupt the oxidation chain by donating a hydrogen ion [24]. DPPH test results were shown as \%RSC and there is a direct correlation (as already described above).

\%RSC values of the investigated emulsions with OEWAF were 18.3 \% RSC for EEDS and 24.7 \%RSC for EMDS (Fig. 3). Placebo samples showed the lowest value of \%RSC, as we expected, $1.85 \%$ RSC for PLE and 6.0 \%RSC for PLM (Fig. 4). Similar results were obtained from Tasić-Kostov et al. [20], who used the same emulsifiers. Better AA was shown by emulsions with a biodegradable emulsifier compared to the samples with conventional Emulgade SE, as we expected. So, these emulsions had a satisfactory AA and a good safety profile. In addition, the results of our study confirmed the benefit of both types of emulsions, stabilized with both types of mixed emulsifier to be carriers of oil extracts of wild apple fruit in a concentration of $6 \%$, in terms of antioxidant efficiency of cosmetic active ingredients.

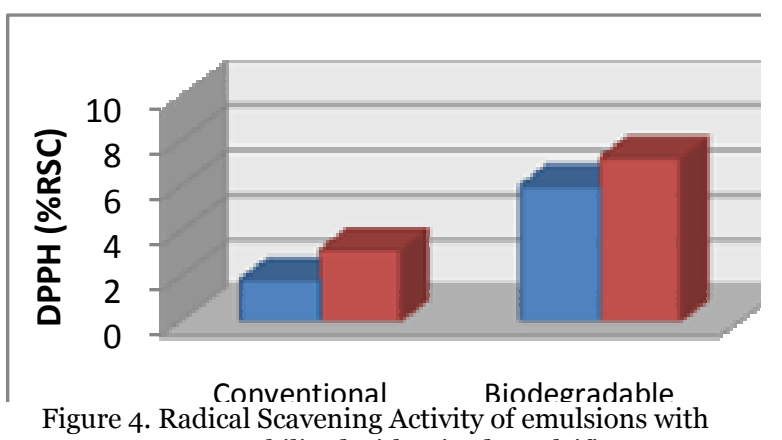

OEWAF, stabilized with mixed emulsifiers

The oil extract after incorporation in both investigated carriers maintained its AA, but less than through direct investigation of extracts only. The obtained results using DPPH test in this study indicate that, most likely, some form of interaction occurred between oil extract and used emulsifier, or structures that stabilize emulsions (bearing in mind that these emulsifiers stabilize emulsion systems by making of complex lamellar structure), which, probably, lead to significant inhibition of the activity of this antioxidant (oil extract of wild apple fruit - S4) after the incorporation in above mentioned carriers [14]. It has been shown that emulsions containing antioxidants show reduced value of oxidative reactions in the experimental system, versus expected, if AS has not been properly dissolved previously. Although in this study pre-dissolution was done, (for the full release of the tested antioxidants from the structures within the emulsion [27]), one assumption could be that the other reason for the reduced AA of oil extract (EMDS) into emulsions was the inadequate pre-dissolution of AS; the molecules of oil extract remained "trapped" into complex structures that stabilize the emulsion, which all resulted in a lower degree of experimentally obtained AA. If this is indeed the case, the question is, in which extent such a "trapped" oil extract (EMDS), as an active substance in preparation, can effectively protect the skin against OS, after the application of the 
emulsion with extract. Another explanation might be that oil can isolate polyphenolic compounds from the WAF well, but it is possible that after extraction, oils can emulsify antioxidant compounds, so they readily release them later, and polyphenols cannot demonstrate their full AA. All this leaves place for further research on this topic.

\section{CONCLUSIONS}

Oil extracts of wild apple fruit showed a good content of polyphenols (which represent a vast array of antioxidant compounds) and satisfactory antioxidant activity. Polyphenols from wild apple fruit can react with free radicals and prevent skin damages. Oil extracts of wild apple fruit, because of the good content of these compounds, may be considered as a great potential for use as active substances in sunscreens, representing feasible alternatives to synthetic ones. Emulsion with oil extract (obtained by digestion with sunflower oil as solvent) stabilized with natural, biodegradable emulsifier (which is safer and better for use) showed the best antioxidant activity. Oil extracts of wild apple fruit, therefore, can be used as active substances in dermocosmetic and/or cosmetic UV protection preparations for health improvement and prevention of UV-related-chronic diseases.

Acknowledgement: Serbian Ministry of education, science and technological development supported this study (Project No III 45017).

\section{REFERENCES}

1. D. Stojiljković, D. Pavlović, I. Arsić, "Oxidative stress, skin aging andantioxidant therapy," Acta Facult. Medicae Naissensis, vol. 31, no. 4, pp. 207-217, 2014.

2. A. Filip et al., "The effects of grape seeds polyphenols on SKH-1 mice skin irradiated with multiple doses of UVB," J. Photochem Photobiol B., vol. 105, no. 2, pp. 133142, 2011.

3. L. Primavesi, M. Piantanida, V. Pravettoni, "Studying Tea Polyphenols and Their Protective Effects on Skin," in: Polyphenols in Human Health and Disease, Italy, chapter 66, pp. 849-858, 2014.

4. Y. Miyachi, "Photoaging from an oxidative standpoint," J. Dermatol Sci, vol. 9, no. 2, pp. 79-86, 1995.

5. D. Stojiljković, I. Arsić, V. Tadić, „Extracts of Wild apple fruit (Malus sylvestris (L.) Mill., Rosaceae) as a source of antioxidant substances for use in production of nutraceuticals and cosmeceuticals," Ind. Crop Prod., vol. 8o, pp. 165-176, 2016.

6. C. A. Elmets et al, "Cutaneous photoprotection from ultraviolet injury by green tea polyphenols," J. Am. Acad. Dermatol., vol. 44, no. 3, pp. 425-432, 2001.

7. G. Xing-Hua, Z. Lan, W. Yan, L. Yuan-Hong, C. HongDuo, "Green Tea Polyphenols and Skin Protection: An Overview," in: Tea in Health and Disease Prevention, China, 2013, chapter 78, pp. 923-931

8. I. Jakšić et al., "Compounding of a topical drug with prospective natural surfactant-stabilized pharmaceutical bases: Physicochemical and in vitro / in vivo characterization - A ketoprofen case study," Eur. J. Pharm. Biopharm., vol. 8o, pp. 164-75, 2012.

9. J. Tucakov, "Lečenje biljem," Beograd, Srbija: Rad, 1997 (J. Tucakov, "Plants treatment," 7th ed., Belgrade, Serbia: Rad, 1997)

10. "Lekovite biljke SR Srbije," ur. M. Sarić, Beograd, Jugoslavija: SANU, 1989 ("Medicinal Plants of SR Serbia,” Ed. M. Sarić., Belgrade, Yugoslavia: SASA, 1989)
11. M. Kalinowska, A. Bielawska, H. LewandowskaSiwkiewicz, W. Priebe, W. Lewandowski, "Apples: content of phenolic compounds vs. variety, part of apple and cultivation model, extraction of phenolic compounds, biological properties," Plant Physiol. Biochem., vol. 84, pp. 169-188, 2014.

12. A. Žugić et al., "Antioxidant activity and phenolic compounds in 10 selected herbs from Vrujci Spa, Serbia," Ind. Crops Prod., vol. 52, pp. 519-527, 2014.

13. I. Arsić et al., "Estimation of dermatological application of the creams with St. John's Wort oil extracts," Molecules, vol. 17, pp. 275-294, 2012.

14. D. Stojiljković et al., "Investigation of effects of different emollients on the structure and skin moisturizing potential of the cosmetic creams," Acta Facult. Medicae Naissensis, vol. 30, no. 4, pp. 193-200, 2013.

15. I. Arsić et al., "Hypericum Perforatum L. Hypericaceae/Guttiferae Sunflower, Olive and Palm Oil Extracts Attenuate Cold Restraint Stress - Induced Gastric Lesions," Molecules, vol. 15, no. 10, pp. 66886698, 2010.

16. A. Žugić et al., "Alkyl polyglucoside - stabilized emulsion as a prospective vehicle for Usnea barbata $\mathrm{CO}_{2}$ supercritical extract: assessing stability, safety and efficiency of a topical formulation," Hem. Ind., Vol. 69, no. 6 , pp. 703-712, 2015.

17. S. Savić, S. Tamburić, M. Savić, "From conventional towards new - natural surfactants in drug delivery systems design: current status and perspectives," Expert Opin Drug Deliv., vol. 7, pp. 353-369, 2010.

18. "Farmakopeja SFRJ", Beograd, Jugoslavija: Savezni zavod za zdravstvenu zaštitu, 1984. ("Pharmacopoea Jugoslavica” Belgrade, Yugoslavia: Federal Institute for Health Protection, 1984.)

19. D. Stojiljković, I. Arsić, M. Tasić-Kostov, "The influence of polar and non-polar emollients on the structure and skin moisturizing potential of the emulsions stabilized by mixed emulsifier," Acta Medica Medianae, vol. 55, no. 2, pp 25-30, 2016

20. M. Tasić-Kostov, "Dermocosmetic emulsions stabilized with lamellar liquid-crystalline phase as a vehicle for lactobionic acid - colloidal structure, efficacy and safety assessments," Faculty of Pharmacy, University of Belgrade, Serbia, Doctoral dissertation. 2013, (in Serbian).

21. V. L. Singleton, J.A. Rossi, "Colorimetry of total phenols withphosphomolybdic phosphotungstic acid reagents," Am. J. Enol. Vitic., vol. 16, no. 3, pp. 144158,1965 .

22. W. Brand-Williams, M.E. Cuvelier, C. Berset, "Use of a free radical method to evaluate antioxidant activity," LWT-Food Sci. Technol., vol. 28, no. 1, pp. 25-30, 1995.

23. H. M. Gordon, P. Maisuthisakul, "Antioxidant and tyrosinase inhibitory activity of mango seed kernel by product," Food Chem., vol. 117, pp. 332-341, 2009.

24. K. Carbone, B. Giannini, V. Picchi, R. Lo Scalzo, F. Cecchini, "Phenolic composition and free radical scavenging activity of different apple varieties in relation to the cultivar, tissue type and storage," Food Chem., vol. 127, no. 2, pp. 493-500, 2011.

25. C. Cekic, M. Ozgen, "Comparison of antioxidant capacity and phytochemical properties of wild and cultivated red raspberries (Rubus idaeus L.)" J. Food Comp. Anal., vol. 23, no. 6, pp. 540-544, 2010.

26. M. Amzad Hossain, S. M. Salehuddin, M. J. Kabir, S. M. M. Rahman, H. P. Vasantha Rupasinghe, "Sinensetin, rutin, 3-hydroxy-5,6,7,4-tetramethoxyflavone and rosmarinic acid contents and antioxidative effect of the skin of apple fruit," Food Chem., vol. 113, no. 1, pp. 187-190, 2009.

27. V. Di Mambro, M. Fonseca, "Assays of physical stability and antioxidant activity of a topical formulation added with different plant extracts", J. Pharm. Biomed. Anal., vol. 37, pp. 287-295, 2005 